\title{
When Twins Collide: Twin Junctions in Nanocrystalline Nickel
}

\author{
Spencer L. Thomas ${ }^{\mathrm{a}}$, Alexander H. King ${ }^{\mathrm{b}}$, David J. Srolovitz ${ }^{\mathrm{a}, *}$ \\ ${ }^{a}$ University of Pennsylvania, Philadelphia, PA 19104 \\ ${ }^{b}$ Division of Materials Sciences and Engineering, Ames Laboratory (USDOE), Ames, IA 50011, USA
}

\section{Abstract}

We present the results of large-scale molecular dynamics simulations of grain growth in polycrystalline nickel with nanoscale grains. The simulations show that grain growth is accompanied by coherent twin boundary (CTB) generation. As the grains grow, twins collide; such collisions result in twin junctions. We catalog all possible twin junctions and show examples of each from the simulations. These include junctions of 2-4 CTBs with grain boundaries and five-fold twin junctions (penta-twins). We elucidate the mechanisms by which all of these junctions form and their relative frequencies. Penta-twins, which are rare in coarse microstructures, occur frequently in nanocrystalline metals. Their absence in macro-scale samples can be traced to the wedge-disclination character (and, consequently, an elastic energy that diverges with sample size). In the nanocrystalline case, the presence of penta-twins can be traced to this twin collision formation mechanism, which is responsible for their wedge-disclination dipole character (relatively small elastic energy). We demonstrate how all CTB junctions, especially penta-twins, retard grain growth.

\section{Keywords: Five-fold Twins, Annealing Twin, Grain Growth, Molecular Dynamics, Nanocrystalline, Disclination}

\section{Introduction}

While twins are observed in bulk materials, they occur with a higher frequency in nanoscale materials. These twins often occur in configurations unique to these materials. For example, penta-twins have been observed in nanowires $[1,2]$, nanoparticles $[3,4,5]$, and nanocrystalline (NC) metals (polycrystals with nanometer-scale grains) $[6,7]$, but are only rarely seen in bulk or coarse-grained materials [8]. Penta-twins in face centered cubic (FCC) metals consist of five coherent twin boundaries (CTBs) meeting at a line parallel to their common $\langle 110\rangle$ axis. The rarity of penta-twins in bulk materials and their high frequency in nanoparticles and nanowires may be attributed to their wedge disclination character, which produces a strain energy that grows with sample size $R$ as $R^{2}$. In the present study, we catalog and determine the mechanism by which twin junctions (including penta-twins) form during grain growth in NC FCC metals (Ni).

In FCC metals, CTBs are typically the lowest-energy grain boundary (GB); they are $\Sigma 3$ symmetric tilt GBs corresponding to a $60^{\circ}$ rotation about a $\langle 111\rangle$ axis. Twins are "coherent" only if they lie on a $\{111\}$ plane. Using the standard FCC notation in which $\{111\}$ planes have an ABCABC stacking sequence, a CTB can be represented as AB\&BA (the vertical line indicates the CTB location). CTBs are atomically sharp, tend to be nearly

\footnotetext{
*Corresponding author. Tel.: +1 (215) 898-6924

Email addresses: thospe@seas.upenn.edu (Spencer L. Thomas), alexking@ameslab.gov (Alexander H. King),
} srol@seas. upenn.edu (David J. Srolovitz) atomically flat, do not disrupt the normal FCC nearest neighbor bonding geometry, and migrate via the propagation of steps. High CTB densities may lead to large strength enhancements in NC metals [9, 10, 11, 12, 13].

Twinning is generally classified into two categories, deformation and annealing twinning, based on the conditions under which twins form. Deformation twinning is a common mode of plastic deformation in NC metals with low stacking fault energy $[9,14]$. Annealing twins usually form absent an applied stress; several mechanisms for this have been proposed. Jin et al. [15] suggest that annealing twins form chiefly as part of the recrystallization process. Farkas et al. [16] observed twin formation during grain growth. Most evidence suggests three primary avenues for postrecrystallization twinning. The growth-accident mechanism implies twin formation when the perfect $\mathrm{ABCABC}$ $\{111\}$ plane sequence is, as the name suggests, accidentally violated during crystal formation [17, 18, 19]. Annealing twins can also form when two grains, misoriented according to a twin relation, come into contact via grain growth [20]. Finally, a twin may form by decomposing one GB into another plus a twin if doing so reduces the total energy [21, 22, 23].

As discussed above, penta-twins have been observed to form in several classes of nanocrystalline metals; including electrodeposited nickel [24], ball-milled NC metals [25, 26], and materials processed using high-pressure torsion [27]. Zhu et al. [28] proposed a mechanism by which pentatwins form in NC materials by sequential twin formation, where twins of a penta-twin form one after another from the coalescence of stacking faults under the influence of

April 8, 2016 
a large, time-varying stress. Bringa et al. [29] reported penta-twin formation in molecular dynamics (MD) simulations of NC copper in the absence of external stresses. They proposed a mechanism, similar to that of Zhu et al. [28], but where the large stresses were associated with localized internal stress associated with the presence of GBs. More recently, Cao et al. [30] observed penta-twin formation in annealing experiments in the absence of an applied stress. In their MD simulations, they noted several different penta-twin formation mechanisms depending on initial microstructures. They observe that, given the right initial conditions, partial dislocation generation and migration can rotate grains and transform general GBs into twins or combinations of twins and other grain boundaries. However, no general formation mechanism was established.

Evidence suggests that penta-twins that form under the influence of large stresses do so by sequential twin addition; this often results in highly non-uniform angles between CTBs in the penta-twin $[28,29]$. On the other hand, electron microscopy observations by Huang et al. [31] show little variation in twin junction angles at penta-twins (maximum angular difference of $\sim 2.5^{\circ}$ ). They concluded that this implies that the constituent CTBs simultaneously join to form penta-twins rather than being added sequentially. These earlier studies can be divided into (1) experimental observations of penta-twins after they were formed and (2) atomistic simulations of multi-twin junctions performed for either very specific junction geometries or where the number of penta-twins observed was very small. Unfortunately, it was not possible to directly observe penta-twin formation in-situ during the experiments; hence, the operative formation mechanisms were by necessity speculative. Although the simulation studies lead to the identification of penta-twin formation mechanisms, the number of such mechanisms was nearly as diverse as the set of observations themselves (e.g., see [30]).

Our goal is to monitor the formation of a large set of twin junctions during grain growth, catalog them, and clarify the mechanisms by which each class of twin junctions form. Included among these junctions, we especially focus on penta-twins to clarify how they form and why they form preferentially in nanomaterials. To this end, we perform large scale MD studies of grain growth in an FCC NC metal and observed the formation of a large number of twin junctions (including penta-twins ) without any external stresses. These simulations demonstrate that there is a relatively small number of different classes of CTB junctions and penta-twin formation is a common occurrence in CTB-forming materials in the presence of GB migration. Our analysis clarifies the relative abundances of different CTB junctions, why penta-twins are common in NC materials, and the influence of CTB junctions on GB migration.

\section{Simulation Methods}

Large-scale MD simulations of an evolving nanocrystalline nickel microstructure were performed at an elevated temperature to study twin junction formation during annealing. In these simulations, the interactions between $\mathrm{Ni}$ atoms were described using an EAM potential [32] and the simulations were performed using the LAMMPS MD simulation software [33]. The melting point for this potential, $T_{m}=1590 \mathrm{~K}$, was determined using the phase coexistence method [34]. The stacking fault energy is $125 \mathrm{~mJ} / \mathrm{m}^{2}$ (in line with experimental values), but overestimates the CTB energy by $20 \mathrm{~mJ} / \mathrm{m}^{2}$ (i.e., $63 \mathrm{~mJ} / \mathrm{m}^{2}$, as compared to the experimental $43 \mathrm{~mJ} / \mathrm{m}^{2}$ ) [32]. The discrepancy in CTB energy is negligible compared with its value relative to all other relevant GB energies.

The simulations were performed using cubic cells of edge length $L \approx 40 \mathrm{~nm}$ and initial mean grain size $L \approx 4.5 \mathrm{~nm}$; periodic boundary conditions were applied $\left(D=L / N^{1 / 3}\right.$, where $N$ is the number of grains in the simulation cell). The initial microstructures were generated using a continuum grain growth simulation method [35] (Fig. 1a); the commonly used voronoi tessellation approach to generating "polycrystalline" microstructures yields (i) grain size distributions that differ greatly from those seen in grain growth [36], (ii) perfectly flat GBs and (iii) unrealistic triple junction angles. The computational cost of this grain growth method is negligible relative to that of the MD annealing simulations. Once the microstructure was obtained, each grain was populated with atoms arranged in a perfect FCC crystal of randomly-chosen orientation. Atoms significantly closer to one another than the equilibrium $0 \mathrm{~K}$ nearest neighbor distance were removed.

Each initial atomic configuration was first relaxed at $T=0 \mathrm{~K}$ using a conjugate gradient energy minimization. The sample was then annealed for 100 ps at $300 \mathrm{~K}$ using $\mathrm{MD}$ and relaxed again. This approach was used to remove any artifacts of the process that generated the initial configuration (see Fig. 1c). Note that during the relaxation step, a small number of twins are formed (none are present in the as-constructed structure), presumably due to partial dislocation formation and propagation to relieve high local stresses. After this "pre-run" was complete, each structure was annealed in an NPT ensemble (Nosè-Hoover thermostat) for $2.5 \mathrm{~ns}$ with zero external stress at $1350 \mathrm{~K}$, which corresponds to $\approx 0.85 T_{m}$ for this potential (see Fig. $1 \mathrm{~d}$ ). The simulation time was chosen to be long enough to observe significant grain growth, but short enough to avoid individual grains growing to span the simulation cell. The temperature was chosen to be as high as possible to maximize grain growth rates without GB melting. This annealing procedure leads to substantial grain growth. Figs. 1c and $1 d$ indicate the formation of many CTBs and CTB junctions, including penta-twins (e.g. the top-center of Fig. 1d), accompanying GB migration.

As the anneals proceeded, atomic configurations from the simulations were stored at regular time intervals and independently quenched (at a rate of $\sim 3 \mathrm{~K} / \mathrm{ps}$ followed by an energy minimization) for analysis. These simulations were analyzed on the basis of several parameters, including atomic-level stress, centrosymmetry parameter, 


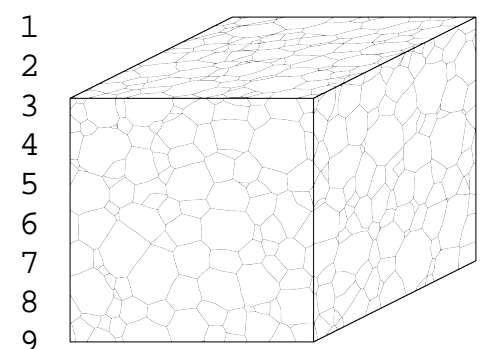

(a)

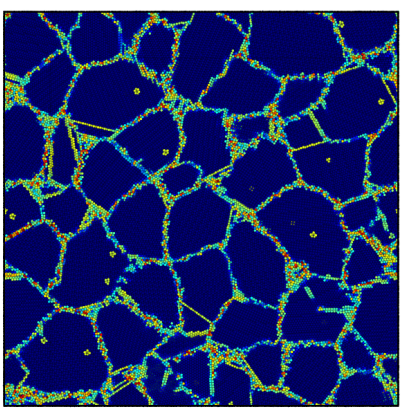

(c) CTBs. hypotheses.

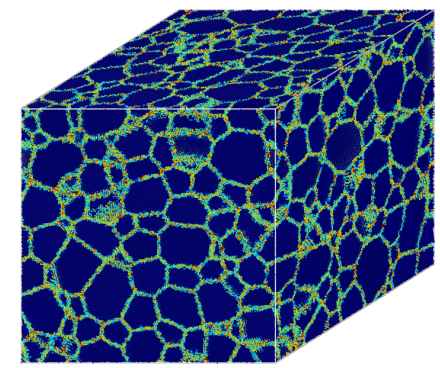

(b)

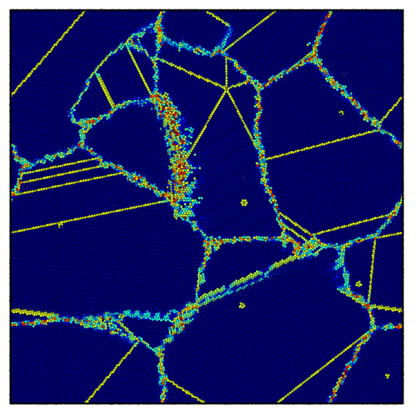

(d)

Figure 1: (a) A sample continuum grain growth simulation microstructure. (b) The same microstructure populated with atoms the initial configuration for an annealing simulation. (c) cross-section of microstructure after initial relaxation (d) cross-section after a 2.5 ns anneal at $0.85 T_{m}$. The atoms in this, and subsequent atomic structures, are assigned a color based upon the centrosymmetry parameter. The straight yellow lines in the cross sections are traces of

and energy density. All visualization was performed using the OVITO atomic visualization software [37]. Additional MD simulations were performed to equilibrate ideal grain boundaries and determine their $T=0 \mathrm{~K}$ energies. Several other ideal configurations were also examined by determine penta-twin energies and test penta-twin formation

The polycrystal annealing simulations were performed on pure samples, whereas in experimental studies, impurities/solutes are always present. This implies that grain growth in the simulations occurs at a much higher rate (lower activation energy for GB migration) than in most experiments where solute drag/segregation is inevitable (with a correspondingly much higher activation energy for GB migration). The difference in kinetics between simulations and experiments may affect the degree to which local equilibrium is obtained in the two cases.

\section{Coherent Twin Boundaries, Junctions and Penta-Twins}

Before discussing CTB junction formation in detail, we digress to review several crystallographic, geometric, and energetic features of CTBs, CTB junctions and the disclination character of penta-twins that will be useful in our analysis. In FCC metals, coherent twin boundaries (CTBs) lie on $\{111\}$ planes and correspond to a $60^{\circ}$ or $120^{\circ}$ twist about the $\langle 111\rangle$ axis normal to their planes. The geometry of the FCC lattice implies that CTBs $(\{111\}$ planes) may be parallel to one another or may intersect along $\langle 110\rangle$ directions at angles of $\theta_{A}=\cos ^{-1}(1 / 3) \approx$ $70.53^{\circ}$ or $\theta_{B}=\cos ^{-1}(-1 / 3) \approx 109.47^{\circ}$ (see Fig. 2 ). Since each CTB rotates the crystal by $2 \pi / 6=60^{\circ}$, a pair of parallel CTBs rotates it by $2 \pi / 3=120^{\circ}$, which corresponds to the three-fold rotational symmetry of an FCC crystal about $\langle 111\rangle$. This $60^{\circ}$ twist about $\langle 111\rangle$ used to create the CTB is identical to a $\theta_{A}$ (symmetric) tilt about $\langle 110\rangle$.

While coherent twin boundaries have a very low energy compared to more general GBs (e.g., the CTB energy in Ni is $63 \mathrm{~mJ} / \mathrm{m}^{2}$ with the potential used in this study, while a $\Sigma 5(310)$ symmetric tilt GB has an energy of $1469 \mathrm{~mJ} / \mathrm{m}^{2}$ ), an incoherent twin boundary has an energy much closer to a general GB than a CTB. Because the energy cost is so high to rotate a CTB away from a $\{111\}$ plane, CTBs tend to remain flat (although they may contain well separated steps). Hence, if a CTB terminates on a GB and that GB is migrating, the CTB simply gets longer or shorter depending on which way the GB migrates. Such a GB may migrate to decrease the grain boundary area in the system (curvature or capillarity driven growth) or for some other reason (e.g., driven by strain energy differences).

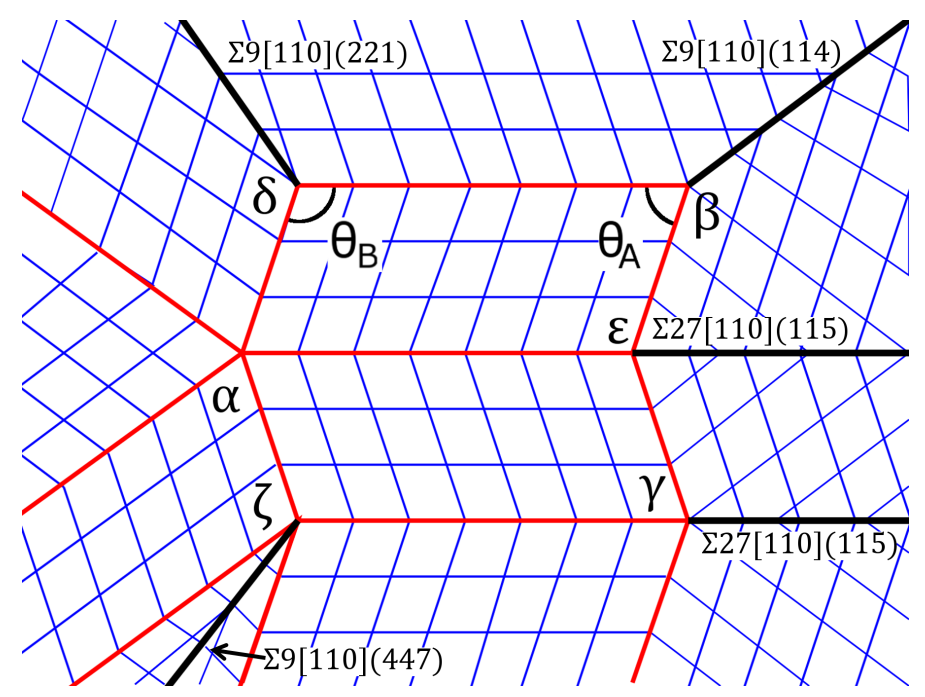

Figure 2: A schematic illustration of a microstructure with different types of twin and twin/grain boundary junctions (labeled with Greek letters). Heavy black lines represent symmetric tilt grain boundaries (the GB bicrystallography is labeled). Red lines represent CTBs. Blue lines represent $\{111\}$ planes.

While it is possible for two non-parallel CTBs to meet and terminate along $\langle 110\rangle$ within a grain interior. However, this would imply a very large distortion of the crystal, corresponding to a lattice rotation of $\pi-2 \theta_{A}$ or $2 \theta_{B}-\pi$ (both equal $\sim 38.94^{\circ}$ ). The elastic energy associated with such a large lattice distortion would be prohibitive. On the other hand, if five, non-parallel CTBs were to meet along a common $\langle 110\rangle$ direction to form a penta-twin, the associated lattice distortion would be relatively small (see junction $\alpha$ in Fig. 2 - the lattice distortion is not shown). 


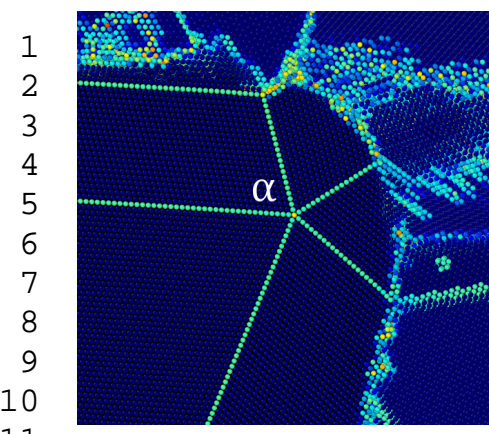

11 (a)

12

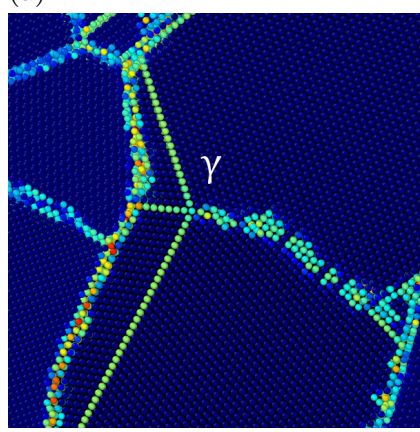

(c)

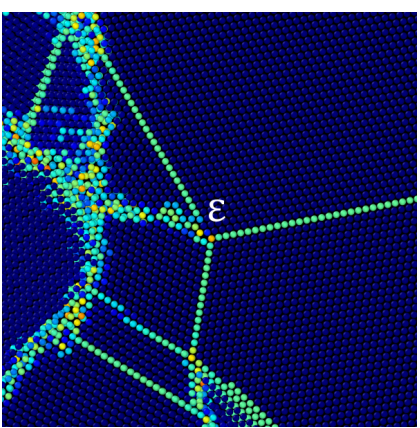

(e) Fig. 2.

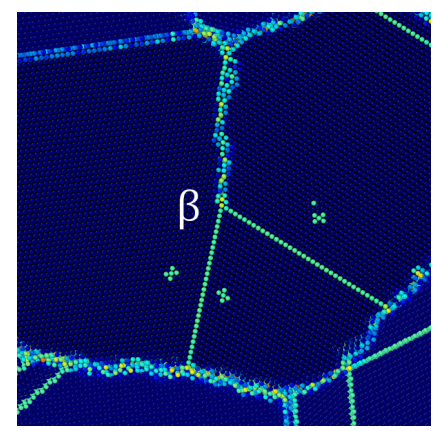

(b)

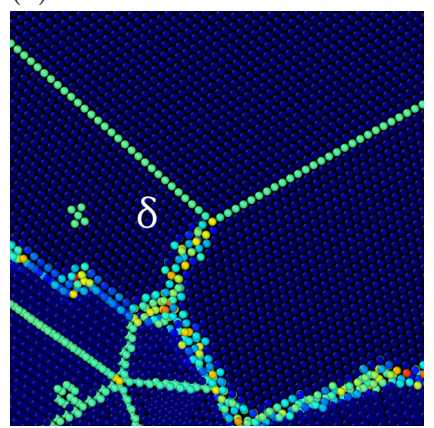

(d)

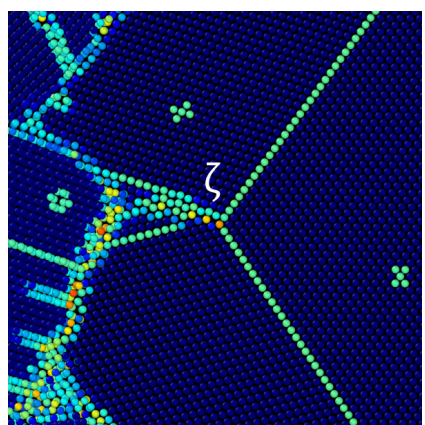

(f)

Figure 3: Observations of different types of twin junctions observed in the MD grain growth simulations. The labels are as shown in

Because $\theta_{A}$ is close to $2 \pi / 5=72^{\circ}$, the penta-twin lattice distortion rotation is $\omega=2 \pi-5 \theta_{A} \approx 7.356^{\circ}$ ). This lattice rotation is a violation of the rotational symmetry of the crystal lattice. Such a symmetry violation represents a defect in the crystal; a wedge disclination. For our purposes, we can think of a wedge disclination formed by removing a wedge of angle $\omega$ from a cylinder and deforming it to bring the faces of the wedge into contact to enforce compatibility. The disclination is a line defect at the wedge apex. Clearly, all junctions of CTBs have disclination character and because $\omega$ is smallest for the penta-twin, the elastic penalty for producing a penta-twin is far smaller than for any other junction between CTBs.

The energy (per unit length) of a wedge disclination $U_{\triangle}$ diverges with the size of the system in which it is embedded. However, this energy is finite if the disclination is confined within a finite material. The classic example of this is the wedge disclination in a cylinder - which can be directly applied to the case of nanowires. In the isotropic elastic limit, a wedge disclination of strength $\omega$ in a cylindrical elastic body of radius $R$ and height $l$ has the following stresses and elastic energy [38]:

$$
\begin{aligned}
& \sigma_{r r}=D \omega \log \left(\frac{r}{R}\right) \quad \sigma_{\theta \theta}=D \omega\left(\log \left(\frac{r}{R}\right)+1\right) \\
& U_{\triangle}=\frac{D \omega^{2} R^{2}}{8} \quad D=\frac{\mu}{2 \pi(1-\nu)}
\end{aligned}
$$

where $\mu$ and $\nu$ are the shear modulus and Poisson ratio. Because the elastic energy grows as $\omega^{2} R^{2}$, disclinations are very high energy defects when the cylinder has a large radius. In a solid, disclinations can screen one another; hence, we can view $R$ as a screening length. In a polycrystal, the elastic energy can be greatly reduced if the grain boundaries can slide (i.e., are surface that can support no shear). Hence, while penta-twins may have very high energy in bulk materials, in nanocrystalline materials (with slipping GBs) or nano-particles/wires this energy can be viewed as the size of the grain or size of the particle/wire. Not surprisingly, penta-twins are most commonly observed in nano-scale materials [39].

\section{Observations From Grain Growth Simulations}

During a $2.5 \mathrm{~ns}$ anneal at $0.85 T_{m}$, the mean grain size grows from $\sim 4.5 \mathrm{~nm}$ to $\sim 9.0 \mathrm{~nm}$, i.e., the mean grain volume grows by nearly one order of magnitude (see Fig. 1). A wide range of defects form spontaneously during grain growth, including vacancies, stacking faults, twin boundaries, and dislocations. As seen in Fig. 1b, the number of CTBs formed during grain growth is large, even though there was no externally applied stress. These are annealing twins. (The mechanisms by which CTBs, dislocations, and vacancies form during grain growth are interesting and widely discussed, but are not the focus of this paper and will not be discussed here.) Sometimes, a single CTB will form; such isolated CTBs typically form by splitting off from a GB, changing the misorientation across the GB. This occurs presumably because the energy of the original grain boundary is higher than the combined energy of the CTB and the new GB (recall that compared with most GBs the CTB energy is negligible). We also note, however, that local stresses may play an important role. In some cases, the twins form as part of an array of parallel CTBs spaced as little as $0.5 \mathrm{~nm}$ apart (see e.g., the grain on the left side near the mid-line of Fig. 1c).

While the formation of such a CTB array may arise as a result of growth accidents behind a migrating grain boundary, we consistently observe such arrays behind migrating triple junctions. The preferential formation of CTBs at triple lines is consistent with recent experimental observations of Lin et al. [40], but while they present a convincing energy argument for single-CTB formation, it does not explain the multiple twin formation we observe. We suspect that there must be an additional contribution to the energy, perhaps a strain energy induced by the triple junction 


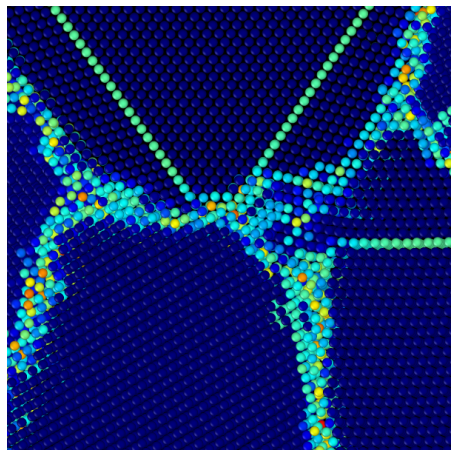

(a)

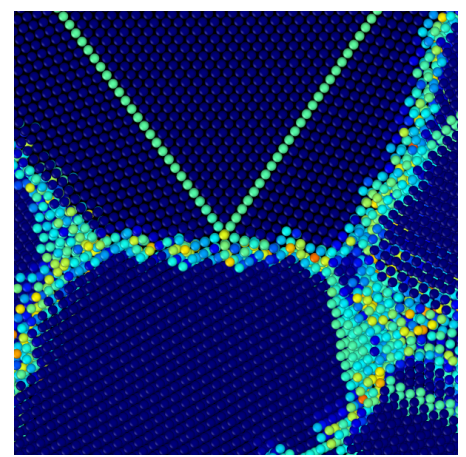

(b)

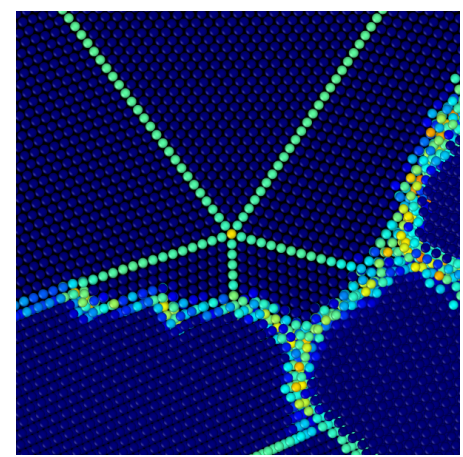

(c)

Figure 4: Formation of a penta-twin by the collision of two CTBs taken from a polycrystal anneal.

that is accommodated by the CTB. If the energy relaxed by this stress accommodation exceeds the formation barrier as well as any change to the total GB interfacial energy, twin formation may be favored. As a result, the CTB concentration is not only high, but also nonuniform.

Significant GB migration in a microstructure with a high density of CTBs leads to other phenomena. While individual CTBs within a grain may be parallel to one another, it is three times more likely that they will be inclined with respect to each other. If two non-parallel CTBs intersect a common GB that migrates toward their line of intersection, they may collide. Such collisions happen frequently in these MD anneals. Such a collision must end in one of two ways. If the GB has insufficient driving force to continue migrating, it will be pinned by the (two GB - two twin) junction (see below). However, if the driving force for GB migration is sufficiently large (this is the more common case), it will continue to migrate leaving a twin junction inside the growing grain. There is a diverse, but finite family of such line junctions. All such line junctions lie along a $\langle 110\rangle$ direction and are illustrated in Fig. 2. There is only one such multiple CTB junctions that also does not also incorporate a GB; namely, the penta-twin (labeled $\alpha$ in Fig. 2). There are five additional multiple CTB junctions that also incorporate a GB (labeled $\beta, \gamma, \delta, \epsilon$ and $\zeta$ in Fig. 2). All such junctions are observed during the course of the grain growth MD simulations; Fig. 3 shows examples of each. We note that the penta-twin commonly forms, despite having wedge disclination character.

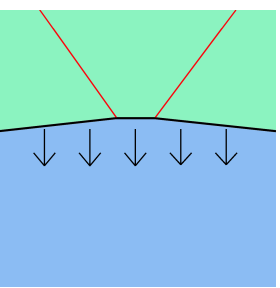

(a)

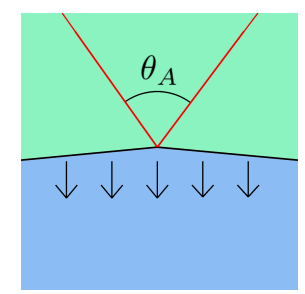

(b)

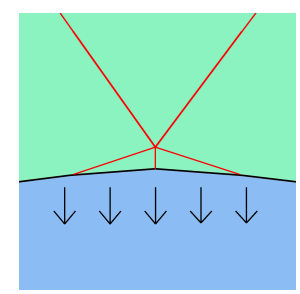

(c)
Figure 5: Schematic of penta-twin formation by the collision of two CTBs (red) at a migrating grain boundary (black).

\section{Penta-twin Formation}

The nature of MD simulations allows us to trace any junction backward in time to establish the means by which it formed. Each penta-twin observed was formed by essentially the same mechanism; an example of this from the annealing simulations is shown in Fig. 4. Two nonparallel CTBs (making an angle $\theta_{A} \approx 70.5^{\circ}$ with respect to one another) in the same grain intersect a single migrating GB (i.e., 2 two-GB/CTB triple junctions), as seen in Fig. 4(a). As the GB migrates, the two CTBs (or two triple junctions) collide, temporarily creating a 2-CTB/two-GB quadra-junction (Fig. 4(b)). For the grain boundary to continue migrating away from this junction, the system must accommodate the difference in orientation in the grain containing the twins. This can be accomplished by adding three new twins, leading to the formation of a penta-twin behind the escaping GB, as seen in Fig. 4(c). This scenario is illustrated in the schematic in Fig. 5.

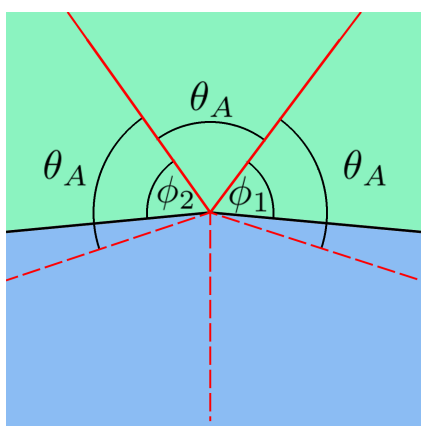

(a)

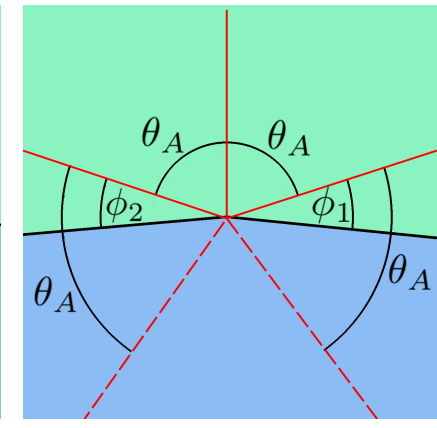

(b)
Figure 6: Schematic illustrating the conditions under which pentatwin formation occurs by the collision of two CTBs (solid red) at a migrating grain boundary (solid black). The red dashed lines indicate the location where three additional CTBs will form after the GB propagates further downward.

Not every CTB collision at a migrating GB (Fig. 5) produces a penta-twin. This can be understood by reference to Fig. 6(a). In order to form the three additional CTBs necessary to create the penta-twin, the new CTBs must lie in the grain that is being consumed by the motion of 

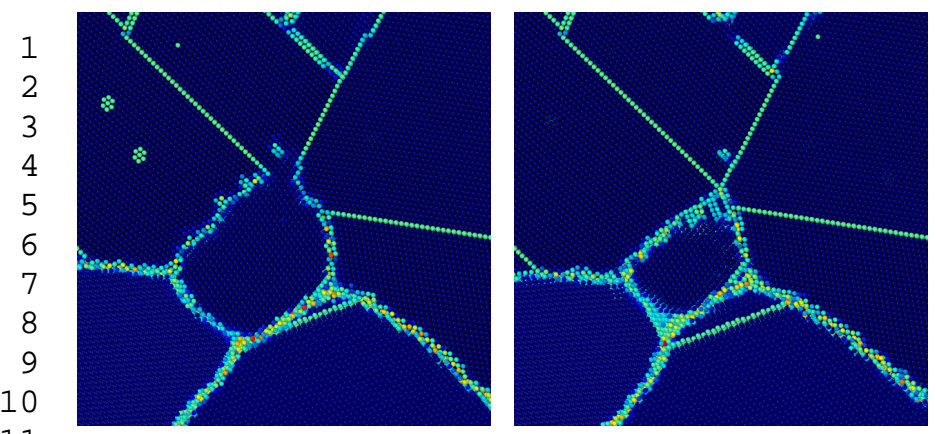

(a)

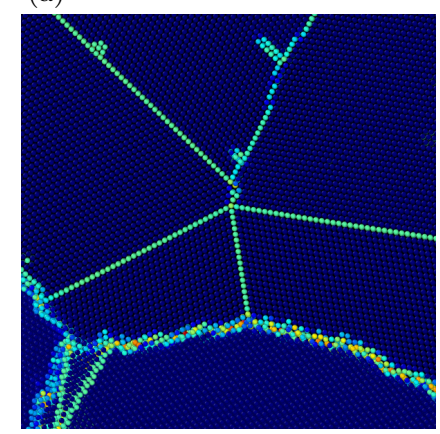

(c)
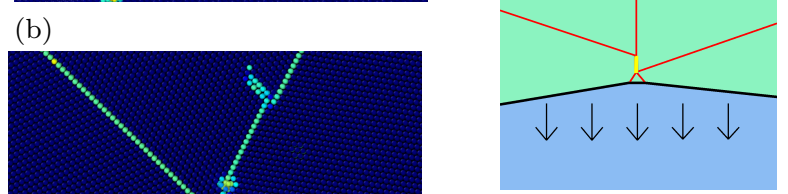

(d)

(a)

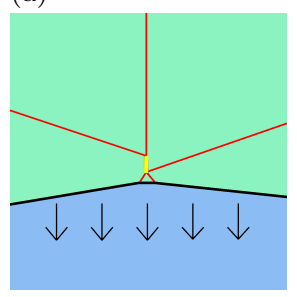
is removed by the step.

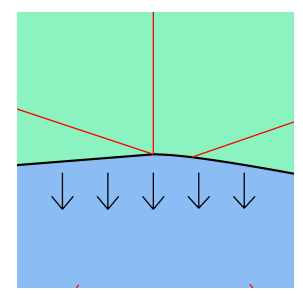

(b)

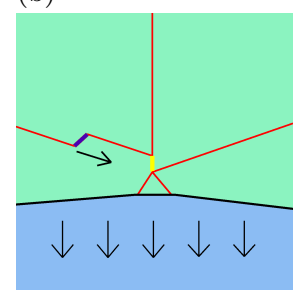

(e)

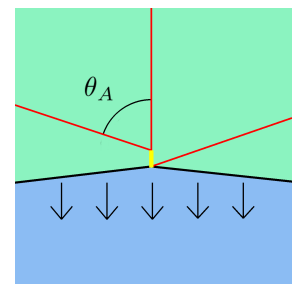

(c)

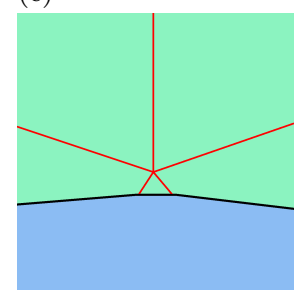

(f)

Figure 8: Schematic illustration of penta-twin formation by the (near) collision of three CTBs (red) at a migrating grain boundary (black), followed by perfection of the penta-twin junction by propagation of a step along one CTB (purple). The yellow line represents the grain boundary segment between the twin junctions that

in Figs. 8(d)-(f). Such perfection of the penta-twin core may be aided by the long range elastic field associated with wedge disclination dipole.

Just as in the two CTB case, not all 3-CTB collisions produce penta-twins. Referring to the schematic in Fig. 6(b), we see that the new CTBs (dashed lines in the schematic) must lie in the grain being consumed by the motion of the GB. This condition can be written as $0<\phi_{1}<\theta_{A}$ and $0<\phi_{2}<\theta_{A}$. We could rewrite the condition for the case of a nearly flat GB, but this assumption is inconsistent with the simulation results in Fig. 7. In the case of a locally flat GB, this condition should be met by construction and is only observed to fail in cases of extreme boundary curvature, such as the boundary of a grain being annihilated by grain growth.

\section{Multiple CTB-GB Junctions}

In addition to the junctions of five CTBs (penta-twins) discussed above, we also observed several other types of CTB junctions, as summarized in Fig. 2. For the case of CTBs meeting at angle $\theta_{A}$, these junctions include two or three CTBs in conjunction with a grain boundary (junctions $\beta$ and $\gamma$ ). For the case of CTBs meeting at angle $\theta_{B}$, these junctions include two, three or four CTBs plus a grain boundary (junctions $\delta, \epsilon$ and $\zeta$ ).

To understand why such multiple CTB/GB junctions form, we first return to the case where two non-parallel CTBs intersect a migrating GB (we ignore the penta-twin formation case here). For the GB to continue to migrate, a new GB must form, as shown schematically in Fig. 9. This explains the formations of junctions $\beta$ and $\delta$ in Fig. 2 where we see a junction of two CTBs and a GB (the two 
cases differ in the angles between the CTBs; $\theta_{A}$ and $\theta_{B}$, respectively). The GB must be present to account for the different orientations of the two grains brought into contact at the two CTB junction. While it is, in principle, possible for the GB to pull away from the two CTB junction in Fig. 9b without forming an additional GB as in Fig. 9c, but this would require the formation of a very strong disclination dipole $\left(\omega=2 \pi-\theta_{A}\right.$ or $\left.2 \pi-\theta_{B}\right)$ with a concomitantly high elastic energy. Hence, formation of multiple CTB-GB junctions are inevitable. Both cases $\beta$ and $\delta$ (Fig. 2) result in the formation of a GB with a misorientation $\Sigma 938.94^{\circ}\langle 110\rangle$. If these GBs are symmetric with respect to the two CTBs meeting at the junction, their boundary planes will be $\{221\}$ and $\{114\}$, respectively. (a)

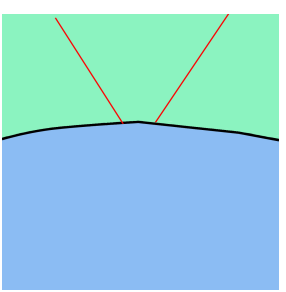

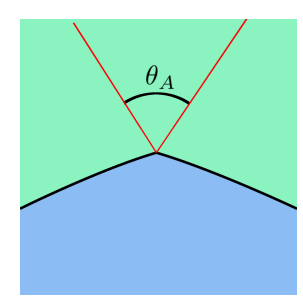

(b)

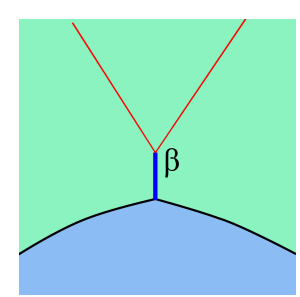

(c)
Figure 9: Schematic of $\beta$ junction formation by the collision of two CTBs (red) at a migrating grain boundary (black). The collision results in a new GB (blue).

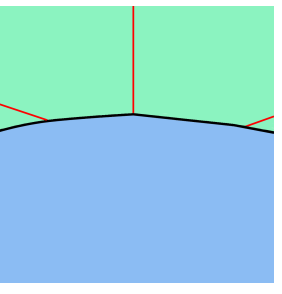

(a)

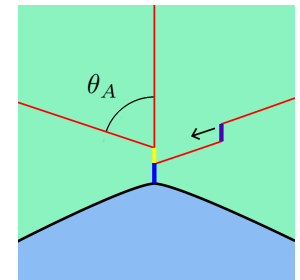

(b)

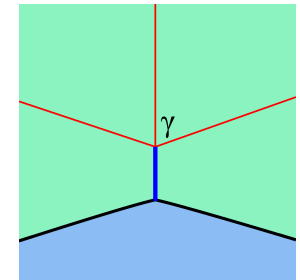

(c)
Figure 10: Schematic of $\gamma$ junction formation by the collision of three CTBs (red) at a migrating grain boundary (black). The collision results in a new GB (blue). (b) shows how a defected $\gamma$ junction can be repaired by the propagation of steps (purple) along a CTB (note that the GB indicated in yellow has a different misorientation than that in blue.

The $\zeta$ junction in Fig. 2 is closely related to the $\delta$ junction; it forms when two CTBs at an angle $\theta_{B}$ meet at a moving GB. The more complex $\zeta$ junction forms simply because the $\Sigma 938.94^{\circ}\{221\}\langle 110\rangle$ GB energy in the $\delta$ junction is nearly $200 \mathrm{~mJ} / \mathrm{m}^{2}$ higher than the GB energy in the $\zeta$ case even when the energies of the two extra CTBs, as shown in Table 1. Hence, when the GB triple junction pulls away from the two CTB junction (see Fig. 9), the $\Sigma 938.94^{\circ}\langle 110\rangle$ that forms, quickly decomposes into a $\Sigma 9$ $27.27^{\circ}\langle 110\rangle$ and two CTBs.

Just as penta-twins can form where two (Fig. 5) or three CTBs (Fig. 8) meet at a migrating GB, so too two or three CTBs meeting at a migrating GB can lead to a multiple CTB/GB junction. While we discussed the case of two
CTBs above with respect to junctions $\beta$ and $\delta$ (and $\zeta$ ), we now turn to the three CTB cases. This case, shown schematically in Fig. 10 for the $\gamma$ junction, is nearly identical to the two CTB case (Fig. 9). In this case, however, since three CTBs meet at the junction, the resultant GB is a $\Sigma 27$ rather than a $\Sigma 9 \mathrm{~GB}$. The same process that led to the formation of the $\gamma$ junction $\left(\theta_{A}\right)$ applies to the $\epsilon$ junction $\left(\theta_{B}\right)$, except that since $2 \theta_{B}>\pi$, the migrating boundary must be strongly curved.

Since the two CTB or three CTB $\theta_{A}$ junction cases can result in the formation of penta-twins, the condition to form the two CTB or three CTB $\theta_{A} / \mathrm{GB}$ junctions ( $\beta$ and $\gamma)$ correspond to the failure to satisfy the penta-twin formation conditions, as described in Sec. 5 and Fig. 6.

In order to validate the junction formation mechanisms deduced from the polycrystal simulations discussed in this section, we performed an additional set of simulations where we isolated the junction formation conditions (multiple CTBs and a moving GB) from the additional complexities associated with the full polycrystalline microstructure. This simulation results confirm the mechanisms and junction formation conditions described in this section. One of these simulations is presented in Section A of the Supplementary Material.

\section{Junction Frequencies}

As shown in Fig. 3, all of the junctions shown schematically in Fig. 2 are observed to form during the polycrystal annealing (grain growth) simulations. The frequencies with which the different junctions form during these simulations vary widely, as shown in Table 1 . Specifically, the $\alpha, \beta$, and $\delta$ junctions occur more frequently than $\gamma, \epsilon$, and $\zeta$ junctions. (We note that $\zeta$ junctions can also forms from 2-CTB collisions, but such junctions were observed to have a short lifespan; if the GB migrates in either direction, it is likely to impinge upon one of the CTBs and transforming it into an $\epsilon$ junction.) This is not surprising since the former are junctions that are generated by the intersection of two CTBs with a migrating GB, the latter are all cases requiring three $\mathrm{CTB}$ intersecting the same migrating GB and the probability of a double collision is much higher than a triple collision. We observe $\sim 6.5$ times as many junctions formed from 2-CTB collisions than 3-CTB collisions. While we might expect even a larger ratio than this given how rare 3-CTB collisions should be, propagation of a kink down a CTB can bring that CTB into contact with another CTB junction (see Fig. 10b) leading to the formation of a 3 -CTB/GB junction; e.g., this can transform $\beta$ into $\gamma$ junctions or $\delta$ into $\epsilon$ junctions.

Both $\alpha$ (penta-twins) and $\beta$ junctions occur primarily from 2-CTB collisions, yet penta-twins are observed much less frequently than $\beta$ junctions in the polycrystal simulations (see Table 1). This can be understood by consideration of the 2-CTB collision penta-twin formation condition described in Sec. 5; namely, $\pi-2 \theta_{A}<\phi_{1}<\theta_{A}$ (see Fig. 6). Considering that $\phi_{1}$ lies in the range $0<\phi_{1}<\pi-\theta_{A}$, the 


\begin{tabular}{|c|c|c|c|c|c|}
\hline Junc. & Symmetric GB & $\begin{array}{c}\text { Energy } \\
\left(\mathbf{m J} / \mathbf{m}^{2}\right)\end{array}$ & $\theta$ & \begin{tabular}{|l|}
$\#$ of \\
CTB
\end{tabular} & $\begin{array}{c}\# \\
\text { Obs. }\end{array}$ \\
\hline$\alpha$ & CTB (No GB) & 63 & $\theta_{A}$ & 5 & 25 \\
\hline$\beta$ & $\Sigma 938.94^{\circ}\{114\}\langle 110\rangle$ & 1090 & $\theta_{A}$ & 2 & 50 \\
\hline$\gamma$ & $\Sigma 2731.59^{\circ}\{115\}\langle 110\rangle$ & 1143 & $\theta_{A}$ & 3 & 8 \\
\hline$\delta$ & $\Sigma 938.94^{\circ}\{221\}\langle 110\rangle$ & 1396 & $\theta_{B}$ & 2 & 49 \\
\hline$\epsilon$ & $\Sigma 2731.59^{\circ}\{115\}\langle 110\rangle$ & 1143 & $\theta_{B}$ & 3 & 7 \\
\hline$\zeta$ & $\Sigma 927.27^{\circ}\{447\}\langle 110\rangle$ & 1099 & $\theta_{B}$ & 4 & 5 \\
\hline Pin & - & - & $\theta_{B}$ & 2 & 20 \\
\hline
\end{tabular}

Table 1: Catalog of the six types of junctions shown in Fig. 2, the GB that symmetrically closes the gap between the CTBs, the GB energy, the angle at which the CTBs meet $\theta$, the number of CTBs that are part of the junctions and the number of each junction observed at an annealing time of $2 \mathrm{~ns}$ in five simulation runs (with the same initial and final grain sizes - see Sec. 2). The final row shows the special case, labeled 'pin', of two GBs meeting a CTB junction (not shown in Fig. 2) that occurs when a migrating GB is unable to pull away from a two CTB junction.

fraction of 2-CTB junction cases that form penta-twins should be $\left(\pi-\theta_{A}\right) /\left[\theta_{A}-\left(\pi-2 \theta_{A}\right)\right]=31.6 \%$. We should expect the ratio of the frequencies of $\beta$ junctions to pentatwins to be $(1-0.316) / 0.316=2.16$. Simulations (Table 1$)$ show this ratio to be 2.0 . This small $(7.5 \%)$ discrepancy may be associated with statistical sampling error and the fact that some penta-twins form from 3-CTB collisions.

We expect that, of the 2-CTB collisions, collisions with angles $\theta_{A}$ and $\theta_{B}$ should occur with the same frequency. Therefore, if we assume that penta-twins occur primarily due to 2-CTB collisions (i.e., we exclude the rare $3-\mathrm{CTB}$ collisions) and that 2-CTB $\theta_{B}$ collisions primarily from $\delta$ and $\zeta$ junctions, we expect the ratio between the sum of the $\delta$ and $\zeta$ junction frequencies and the sum of the $\alpha$ and $\beta$ junction frequencies to be approximately equal. In simulations, the actual ratio is 0.72 . However, this fails to account for cases in which a two-CTB collision at angle $\theta_{B}$ fails to form a junction. This is the dominant form of CTBGB pinning, as will be discussed in Sec. 8. There are 20 observed cases of persistent junctions of two CTBs angled at $\theta_{B}$ intersecting two GBs (the 'pin' case in Table 1). These junctions are not observed to be persistent in the $\theta_{A}$ case. Accounting for these junctions, the ratio is $\sim 0.99$, which matches expectations.

We can deduce the energy of different junctions by considering the sum of the energies of all CTBs and a GB that meet at a junction. Imagine drawing a circle centered around an ideal junction from which all CTBs and GBs radiate outward. The total boundary energy in the circle is proportional to the circle radius and the sum of the energy of all of the boundaries radiating out of the junction. The relevant GB and CTB energies are given in Table 1 . The CTB/GB $(\beta-\zeta)$ junction energies range from $1.2 \mathrm{~J} / \mathrm{m}^{2}$ to $1.5 \mathrm{~J} / \mathrm{m}^{2}$, while the penta-twin is only $0.32 \mathrm{~J} / \mathrm{m}^{2}$ (note, here we neglect the junction core energies and return to elastic energy effects below). On the other hand, the frequency of penta-twins is much smaller than that of $\beta$ and $\delta$ junctions. Clearly, the relative frequencies of occurrence of the different junctions is not determined by their energetics. Rather, as described above, these relative frequencies are determined by the junction formation mechanisms; i.e., the frequencies of occurrence are determined by kinematics not thermodynamics. Note that the penta-twins have wedge disclination character and therefore have an associated elastic energy that should be accounted for in this analysis. However, as discussed below in Sec 8, this contribution is very small in grains of the size range sampled in the simulations.

\section{Analysis and Discussion}

The simulations presented above demonstrate that penta-twins form frequently during grain growth in nanocrystalline materials prone to twin formation. This is a surprising result since penta-twins have wedge disclination character (disclination strength $\omega=2 \pi-5 \theta_{A}=$ $7.356^{\circ}$ ) and the elastic energies of disclinations (per unit length) diverge with sample size as $R^{2}$ [41]. While in finite nanoscale materials, such as nanowires and nanoparticles, penta-twins have been observed $[39,1,2,3,4,5]$, pentatwins are rarely observed in macroscopic materials (with the exception of highly deformed metals [8]). The pentatwin energy (per unit length parallel to the junction) in, for example, a nanowire, scales with nanowire radius $R$ as

$$
U_{\triangle}=5 R \gamma_{C T B}+U_{\triangle}=5 R \gamma_{C T B}+D \omega^{2} R^{2} / 8 .
$$

We note, however, that this nanowire solution is not directly applicable to nanocrystalline materials, where the sample is very large even though the grain size is on the nanoscale.

In order to examine the twin junction thermodynamics in a nanocrystalline material we consider three cases, shown in Fig. 11; a single GB, a $\beta$ junction and a pentatwin (along with its associated wedge disclination) as they are formed through GB migration. The difference in energy (per unit length) between a GB and a GB with a nearby $\beta$ junction (Figs. 11 a and $b$ ) is:

$$
\Delta U_{\beta}=2 R \gamma_{C T B}+d \gamma_{G B},
$$

where $\gamma_{G B}$ refers to the energy (per area) of the GB that extends from the junction to the migrating GB. This equation shows that energy of the system increases in proportion to the junction/migrating GB separation. For a penta-twin near a GB (Fig. 11c), a similar analysis of the energy difference yields:

$$
\Delta U_{\triangle^{\prime}}=(2 R+c d) \gamma_{C T B}+U_{2 \triangle}^{e}
$$

where $U_{2 \triangle}^{e}$ is the elastic energy, $d$ is the separation between the penta-twin and the GB, $c d$ is the total length of all of the CTBs that extend from the penta-twin to the GB, and $c=1+2 / \cos (2 \pi / 5) \approx 7.5$. The $c d$ term is analogous to the $d \gamma_{G B}$ term in Eq. 3 . 
In order to analyze the penta-twin elastic energy (Fig. 11c), we first recall how the penta-twin forms: a two CTB junction forms through the migration of a GB upon which the two CTBs impinge, the further migration of which generates three new CTBs converting the two CTB junction into a penta-twin (see Fig. 5). The pentatwin is an $\omega=7.356^{\circ}$ wedge disclination. However, we can think of that disclination as being created by cutting out a wedge of opening angle $\omega=7.356^{\circ}$ the apex of which is at the penta-twin and the base of which is the migrating GB. When this wedge is closed-up to create the disclination (imagine sliding along the GB), the penta-twin is not a single wedge disclination but a wedge disclination dipole, where one disclination sits at the penta-twin and its opposite sits at the GB, a distance $d$ away, as shown in Fig. 11c. This means that the elastic field associated with a penta-twin in a small grain has wedge disclination dipole character, rather than single wedge disclination character. Therefore, the very large (divergent) energy of a disclination in an elastic medium is effectively screened by its opposite counterpart at the GB.

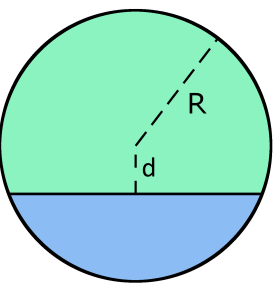

(a)

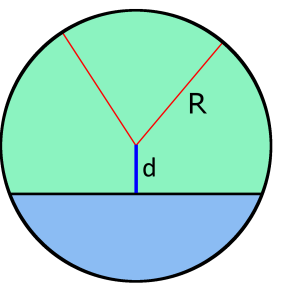

(b)

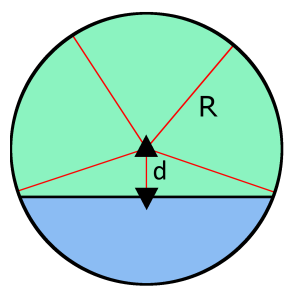

(c)

Figure 11: A cylindrical sample of radius $\mathrm{R}$ with a) a GB displaced $d$ from the center, b) the same GB with a $\beta$ junction centered on the axis, and c) the same GB with a penta-twin centered on the axis and accompanying disclinations. The black triangles represent positive (pointing up) and negative (pointing down) disclinations.

The elastic fields and energy of a wedge disclination dipole $U_{2 \triangle}^{e}$ can be derived from elastic solutions in the literature [42]:

$$
U_{2 \triangle}^{e}=\frac{D \omega^{2} d^{2}}{4}\left[2 \ln \frac{R}{d}+3\right] .
$$

Note, that unlike the elastic energy of a wedge disclination that is proportional to $R$, this divergence is very weak proportional to $\ln R$. This solution resembles the classic solution of an edge dislocation [43] with burgers vector $\omega d$. In the present problem, $d$ is simply the distance between the penta-twin core and the grain boundary, as shown in Fig. 11c. Substituting Eq. 5 for the elastic energy into Eq. 4, we find the energy difference for a penta-twin near a non-slipping or welded GB to be

$$
\Delta U_{\Delta^{\prime}}^{w}=(2 R+c d) \gamma_{C T B}+\frac{D \omega^{2} d^{2}}{4}\left[2 \ln \frac{R}{d}+3\right] .
$$

Interestingly, the penta-twin energy has both a term that is linear in $d$, like for the $\beta$ junction, plus an additional term that scales as $d^{2}$.
An implicit assumption in how we determined the elastic energy of a disclination dipole near a GB, above, is that there is continuity of traction and displacement across the GB (the so-called welded interface condition). There is a growing body of work, however, that suggests that grain boundaries easily slide on the nanometer scale at temperatures as low as room temperature $[44,45,46]$ and that most GBs slide at sufficiently high temperature. We note that while grain boundary sliding may be limited by GB triple junctions, mass transport via Coble creep can relax this constraint; grain rotation rates mediated by this process scale with grain size as $L^{-4}$ [47]. Hence, GB sliding should be especially effective in nanocrystalline materials.

GB sliding modifies the elastic boundary conditions at the GB; i.e., from continuity of all tractions to continuity of normal tractions and zero tangential/shear tractions such that the tangential displacement can jump across the GB. Modifying the elastic field of a disclination dipole near a slipping interface using the elastic fields determined in [48], we find that $U_{2 \triangle}^{e}$ is reduced from $\frac{D \omega^{2} d^{2}}{4}\left[2 \ln \frac{R}{d}+3\right]$ to $\Lambda D \omega^{2} d^{2}$ where we determined $\Lambda=0.597 \approx 3 / 5$ based upon numerical integration (see Section B of the Supplementary Material). Hence, the energy difference of a penta-twin near a slipping grain boundary is:

$$
\Delta U_{\triangle^{\prime}}^{s}=(2 R+c d) \gamma_{C T B}+\Lambda D \omega^{2} d^{2} .
$$

As with the welded GB case, the penta-twin near a slipping GB has terms that are both linear and quadratic in $d$. Critically, however, the elastic energy is finite and has no dependence on $\mathrm{R}$. In reality, the penta-twin energy likely lies between the $\Delta U_{\Delta^{\prime}}^{w}$ and $\Delta U_{\Delta^{\prime}}^{s}$ limiting cases.

Comparing the energies of a $\beta$ junction (Eq. 3) with that of the penta-twin (Eqs. 6 or 7 ) near a GB shows that the penta-twin is lower energy than the $\beta$ junction for $d<d^{*}$ and higher for $d>d^{*}$ where $d^{*}$ is between 1.3 and 3.7 $\mathrm{nm}$ (for $\mathrm{Ni}$, where we assumed that $R \sim 9 \mathrm{~nm}$, the mean grain diameter at the end of each simulation), depending on whether the GB can or cannot slide.

Examination of the MD grain growth simulation results show that CTB junctions tend to slow GB migration/grain growth. We can understand this on the basis of the interaction between a junction and the migrating GB from which it formed. In the $\beta-\zeta$ junction cases, the junctions contain 2-4 CTBs and a new GB, as discussed above. The motion of the migrating GB extends the new GB that radiates from the CTB junction. Since the energy of the new GB is finite (and positive), extending this GB costs energy, and that the total GB energy (product of the GB area and the GB energy/area) is proportional to the distance between the CTB junction and the migrating GB, $d$. Hence, the retarding force on the migrating GB is a constant (independent of the spacing between the migrating GB and the junction) and is roughly proportional to the energy of the GB radiating out from the junction. We can estimate the influence of this extra GB in two dimensions by reference to the von Neumann-Mullins [49, 50] grain 
growth law

$$
\dot{A}=\frac{\pi}{3} M \gamma(n-6),
$$

where $\dot{A}$ is the rate of change of grain area, $M$ is the (isotropic) GB mobility and $\gamma$ is its isotropic energy, and $n$ is the number of grain sides/triple junctions. Adding an extra triple junction yields a change in the rate at which the grain shrinks of

$$
\frac{\dot{A}(n+1)-\dot{A}(n)}{\dot{A}(n)}=\frac{1}{(n-6)}
$$

or $\dot{A}=(\pi / 3) M \gamma(n-5), c f$. Eq. 8. This is a major change in the grain growth law. In three dimensions, a similar effect is expected but is complicated by the fact that the extension of the von Neumann-Mullins law to three dimensions is not purely topological [51].

Unlike the other CTB junctions, a penta-twin does not introduce a GB radiating from the junction to the migrating GB. However, the penta-twin effect on grain growth is potentially stronger than that of the $\beta-\zeta$ junctions. We recall that the energy of the penta-twin in Eqs. 6 and 7 has both a term linear in $d$ and one proportional to $d^{2}$. Interestingly, the retarding force that the penta-twin junction exerts on the migrating GB is proportional to the separation between the junction and the GB, $d$. Hence, pentatwins are spring-like in how they pull the GB back toward the junction - the greater the separation, the larger the retarding force. This implies that migration of a GB away from the penta-twin core will be severely impeded.

Returning to the simulations, we observe a tendency for penta-twins ( $\alpha$ junctions) to be closer to the GBs from which they form then are the other CTB junctions (which tend to be at random locations inside grains). This may be associated with the spring-like retarding force that only penta-twins exert on migrating boundaries. However, our statistics are not sufficient to draw any strong conclusions here.

We note that in the non-penta-twin $(\beta-\zeta)$ junction cases, the formation of the GB at the CTB junction as the original GB migrates away (e.g., see Fig. 9c) also retards GB motion. The larger the energy of the GB that is part of the junction, the greater the retarding force. In some cases, the retarding force is too large for the migrating GB to pull away from the junction leaving a junction of two CTBs and two GBs (labeled "Pin" in Table 1) and shown in Fig. 12. We observe pinning only in the cases where CTBs meet at $\theta_{B}$ and never at $\theta_{A}$. The most common such case is the $\delta$ junction. The GB associated with the $\delta$ junction has a much higher energy than any of the other GBs (see Table 1). This allows us to understand the relative frequencies of the two types $\left(\theta_{A}\right.$ and $\left.\theta_{B}\right)$ of 2 -CTB collision junctions observed junction frequencies reported in Table 1. While we should expect that $\theta_{A}$ CTB collisions occur with equal frequency as the $\theta_{B}$ collisions (based on geometry). 2-CTB collisions at the angle $\theta_{A}$ ( $\alpha /$ pentatwin or $\beta$ junctions) occur 75 times in the simulations (see

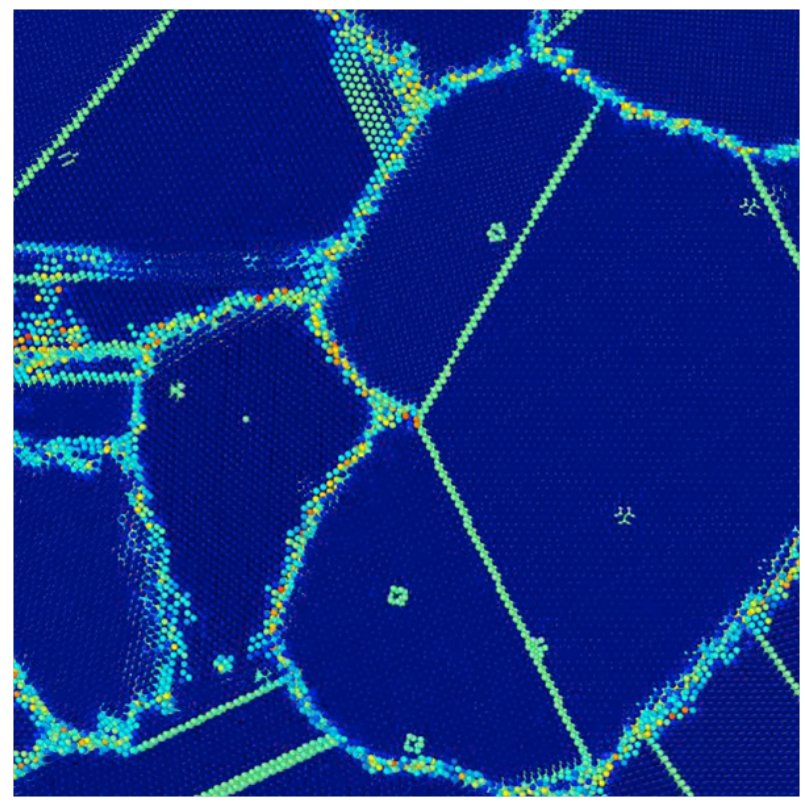

Figure 12: A GB heavily distorted by the pinning influence of a twin junction of type $\delta$. Taken from a polycrystal anneal.

Table 1). On the other hand, 2-CTB collisions at the angle $\theta_{B}$ ( $\delta$ and $\zeta$ junctions $)$ occur 54 times. However, if we include the $\theta_{B}$ "Pin" junctions (20 observations), $\theta_{B}$ junctions is 74 . Hence, we see that retarding forces play an important role in the distribution of junction frequencies.

\section{Conclusions}

We presented the results of a series of large-scale molecular dynamics simulations of grain growth in nanocrystalline nickel. Examination of the evolving microstructure shows that grain growth is accompanied by frequent coherent twin boundary (CTB) generation. The CTBs typically extend across entire grains. Hence, when grain boundaries migrate, CTBs collide and junctions between 2-4 CTBs and grain boundaries readily occur. We catalog all possible such junctions, showed examples of each from the grain growth simulations and deduce the mechanisms by which each type of junction occurs. Based upon simple geometric ideas, we predict the relative frequencies of the different junctions and compare these with data obtained from the simulations.

One particularly interesting class of CTB junctions is the five-fold twin junction; i.e., penta-twin. Penta-twins are rare in materials of large grain size, but common in nanowires and nanoparticles because penta-twins necessarily have wedge-disclination character; the elastic energy of a disclination grows as the square of the sample size. In this context, however, penta-twins (like the other CTB junctions) form by GB migration; the elastic energy of a penta-twin in this case scales like that of a wedgedisclination dipole. This energy is dominated by a term proportional to the square of the spacing between pentatwin and grain boundary and depends only very weakly 
on sample size. Hence, the penta-twin energy is relatively small in nanocrystalline materials and penta-twins readily occur.

All CTB junctions significantly retard continued GB migration/grain growth. For the most common case, where the CTB junctions incorporate a GB, such junctions provide a retarding force that is independent of the distance between junction and migrating boundary. We analyzed the effect of this retardation on grain growth on the basis of the classical von Neumann-Mullins description of grain size evolution. Penta-twins, on the other hand, retard GB migration via a force that increases linearly with CTB junction - migrating GB separation. Hence, pentatwins can have a particularly pronounced effect on grain growth in nanocrystalline materials.

\section{Acknowledgement}

The authors gratefully acknowledge useful discussions with Prof. Kun Zhou and Dr. Hui Feng from the Nanyang Technological University and Prof. Nikolaos Aravas of the University of Thessaly on disclination elasticity and thank Dr. Emanuel Lazar of the University of Pennsylvania for providing Fig. 1a. SLT and DJS at the University of Pennsylvania designed this research, performed and analyzed the computer simulations, and performed the theoretical analysis. Work at the Ames Laboratory was supported by DOE, Office of Science, BES under Contract \# DEAC02-07CH11358. AHK provided geometrical analyses and interpretation of the structures, and contributed to the interpretation of the processes that produced them. This work used the Extreme Science and Engineering Discovery Environment (XSEDE), which is supported by National Science Foundation grant number ACI-1053575 [52].

[1] G. W. Zhou, Z. Zhang, Z. G. Bai, S. Q. Feng, D. P. Yu, Transmission electron microscopy study of si nanowires, Applied Physics Letters 73 (1998) 677-679.

[2] B. Wu, A. Heidelberg, J. J. Boland, J. E. Sader, X. Sun, Y. Li, Microstructure-hardened silver nanowires, Nano Letters 6 (2006) 468-472.

[3] J. Allpress, J. Sanders, The structure and orientation of crystals in deposits of metals on mica, Surface Science 7 (1967) $1-25$.

[4] L. D. Marks, D. J. Smith, High resolution studies of small particles of gold and silver. i. multiply-twinned particles, Journal of Crystal Growth 54 (1981) 425-432.

[5] J. A. Ascencio, M. Prez, M. Jos-Yacamn, A truncated icosahedral structure observed in gold nanoparticles, Surface Science $447(2000) 73-80$

[6] T. Wada, T. Negami, M. Nishitani, Fivefold multiply twinned crystallites in cuinse2, Applied Physics Letters 64 (1994) 333335.

[7] J. Narayan, A. R. Srivatsa, K. V. Ravi, Mechanism of formation of 110 oriented fivefold microcrystallites in diamond films, Applied Physics Letters 54 (1989) 1659-1661.

[8] W. Wang, S. Lartigue-Korinek, F. Brisset, A. L. Helbert, J. Bourgon, T. Baudin, Formation of annealing twins during primary recrystallization of two low stacking fault energy nibased alloys, Journal of Materials Science 50 (2015) 2167-2177.

[9] Y. T. Zhu, X. Z. Liao, X. L. Wu, Deformation twinning in nanocrystalline materials, Progress in Materials Science 57 (2012) $1-62$.
[10] J. Bezares, S. Jiao, Y. Liu, D. Bufford, L. Lu, X. Zhang, Y. Kulkarni, R. J. Asaro, Indentation of nanotwinned fcc metals: Implications for nanotwin stability, Acta Materialia 60 (2012) $4623-4635$.

[11] L. Lu, R. Schwaiger, Z. W. Shan, M. Dao, K. Lu, S. Suresh, Nano-sized twins induce high rate sensitivity of flow stress in pure copper, Acta Materialia 53 (2005) 2169 - 2179.

[12] Z. You, X. Li, L. Gui, Q. Lu, T. Zhu, H. Gao, L. Lu, Plastic anisotropy and associated deformation mechanisms in nanotwinned metals, Acta Materialia 61 (2013) 217 - 227.

[13] R. Ott, J. Geng, M. Besser, M. J. Kramer, M. Wang, E. S. Park, R. LeSar, A. H. King, Optimization of strength and ductility in nanotwinned ultra-fine grained ag: Twin density and grain orientations, Acta Materialia 96 (2015) 378-389.

[14] T. Brown, C. Saldana, T. Murthy, J. Mann, Y. Guo, L. Allard, A. King, W. Compton, K. Trumble, S. Chandrasekar, A study of the interactive effects of strain, strain rate and temperature in severe plastic deformation of copper, Acta Materialia 57 (2009) 5491-5500.

[15] Y. Jin, B. Lin, M. Bernacki, G. S. Rohrer, A. D. Rollett, N. Bozzolo, Annealing twin development during recrystallization and grain growth in pure nickel., Materials Science \& Engineering: A 597 (2014) $295-303$.

[16] D. Farkas, E. Bringa, A. Caro, Annealing twins in nanocrystalline fcc metals: A molecular dynamics simulation, Phys. Rev. B 75 (2007) 184111.

[17] S. Dash, N. Brown, An investigation of the origin and growth of annealing twins, Acta Metallurgica 11 (1963) 1067 - 1075.

[18] H. Gleiter, The formation of annealing twins, Acta Metallurgica 17 (1969) $1421-1428$.

[19] S. Mahajan, C. Pande, M. Imam, B. Rath, Formation of annealing twins in f.c.c. crystals, Acta Materialia 45 (1997) 2633 -2638 .

[20] W. Burgers, J. Meijs, T. Tiedema, Frequency of annealing twins in copper crystals grown by recrystallization, Acta Metallurgica 1 (1953) $75-78$.

[21] R. L. Fullman, J. C. Fisher, Formation of annealing twins during grain growth, Journal of Applied Physics 22 (1951) 1350-1355.

[22] P. J. Goodhew, Annealing twin formation by boundary dissociation, Metal Science 13 (1979) 108-112.

[23] R. Jahn, A. King, Dissociation of grain boundaries induced by changes of composition, the ejection of dislocations from grain boundaries, and the nucleation of diffusion induced grain boundary migration, Acta Metallurgica 40 (1992) 551-558.

[24] C. R. Hall, S. A. H. Fawzi, On the occurrence of multiply twinned particles in electrodeposited nickel films, Philosophical Magazine A 54 (1986) 805-820.

[25] J. Y. Huang, Y. K. Wu, H. Q. Ye, Deformation structures in ball milled copper, Acta Materialia 44 (1996) 1211 - 1221.

[26] X. Z. Liao, J. Y. Huang, Y. T. Zhu, F. Zhou, E. J. Lavernia, Nanostructures and deformation mechanisms in a cryogenically ball-milled al-mg alloy, Philosophical Magazine 83 (2003) 30653075.

[27] X. H. An, Q. Y. Lin, S. D. Wu, Z. F. Zhang, R. B. Figueiredo, N. Gaoc, T. G. Langdon, Formation of fivefold deformation twins in an ultrafine-grained copper alloy processed by highpressure torsion, Scripta Materiala 64 (2011) 249-252.

[28] Y. T. Zhu, X. Z. Liao, R. Z. Valiev, Formation mechanism of fivefold deformation twins in nanocrystalline face-centeredcubic metals, Applied Physics Letters 86 (2005) 103112.

[29] E. M. Bringa, D. Farkas, A. Caro, Y. M. Wang, J. McNaney, R. Smith, Fivefold twin formation during annealing of nanocrystalline cu, Scripta Materialia 59 (2008) $1267-1270$.

[30] Z. Cao, L. Xu, W. Sun, J. Shi, M. Wei, G. Pan, X. Yang, J. Zhao, X. Meng, Size dependence and associated formation mechanism of multiple-fold annealing twins in nanocrystalline cu, Acta Materialia 95 (2015) $312-323$.

[31] P. Huang, G. Q. Dai, F. Wang, K. W. Xu, Y. H. Li, Fivefold annealing twin in nanocrystalline cu, Applied Physics Letters 95 (2009) -.

[32] Y. Mishin, D. Farkas, M. J. Mehl, D. A. Papaconstantopoulos, 
Interatomic potentials for monoatomic metals from experimental data and $a b$ initio calculations, Phys. Rev. B 59 (1999) 3393-3407.

[33] S. Plimpton, Fast parallel algorithms for short-range molecular dynamics, Journal of Computational Physics 117 (1995) 1-19.

[34] G. P. Puna, Y. Mishin, Development of an interatomic potential for the ni-al system, Philosophical Magazine 89 (2009) 32453267.

[35] E. A. Lazar, J. K. Mason, R. D. MacPherson, D. J. Srolovitz, A more accurate three-dimensional grain growth algorithm, Acta Materiala 59 (2011) 6837-6847.

[36] J. K. Mason, E. A. Lazar, R. D. MacPherson, D. J. Srolovitz, Geometric and topological properties of the canonical graingrowth microstructure, Phys. Rev. E 92 (2015) 063308.

[37] A. Stukowski, Visualization and analysis of atomistic simulation data with ovito - the open visualization tool, Modelling and Simulation in Materials Science and Engineering 18 (2010) 015012 .

[38] R. de Wit, Partial disclinations, Journal of Physics C: Solid State Physics 5 (1972) 529.

[39] H. Hofmeister, Fivefold twinned nanoparticles, in: H. Nawla (Ed.), Encyclopedia of Nanoscience and Nanotechnology, American Scientific Publishers, 2004, pp. 431-452.

[40] B. Lin, Y. Jin, C. Hefferan, S. Li, J. Lind, R. Suter, M. Bernacki, N. Bozzolo, A. Rollett, G. Rohrer, Observation of annealing twin nucleation at triple lines in nickel during grain growth, Acta Materialia 99 (2015) $63-68$.

[41] A. E. Romanov, A. L. Kolesnikova, Application of disclination concept to solid structures, Progress in Materials Science 54 (2009) $740-769$.

[42] A. E. Romanov, European research conference on plasticity of materials-fundamental aspects of dislocation interactions: Lowenergy dislocation structures iii screened disclinations in solids, Materials Science and Engineering: A 164 (1993) $58-68$.

[43] F. Nabarro, Dislocations in Solids. Vol. 9: Dislocations and Disclinations, North-Holland, 1992.

[44] T. G. Langdon, Grain boundary sliding as a deformation mechanism during creep, Philosophical Magazine 22 (1970) 689-700.

[45] R. Raj, M. Ashby, Grain boundary sliding, and the effects of particles on its rate, Metallurgical Transactions 3 (1972) 19371942.

46] H. Van Swygenhoven, P. M. Derlet, Grain-boundary sliding in nanocrystalline fcc metals, Phys. Rev. B 64 (2001) 224105.

[47] K. Harris, V. Singh, A. King, Grain rotation in thin films of gold, Acta Materialia 46 (1998) 2623 - 2633.

[48] H. Y. Yu, S. C. Sanday, Disclinations in bimaterials, physica status solidi (a) 126 (1991) 355-365.

[49] J. von Neumann, Metal interfaces, Written discussion on a paper of C. S. Smith, 1952.

[50] W. W. Mullins, Two-dimensional motion of idealized grain boundaries, Journal of Applied Phys. 27 (1956) 900-904.

[51] R. D. MacPherson, D. J. Srolovitz, The von Neumann relation generalized to coarsening of three-dimensional microstructures, Nature 446 (2007) 1053-1055.

[52] J. Towns, T. Cockerill, M. Dahan, I. Foster, K. Gaither, A. Grimshaw, V. Hazlewood, S. Lathrop, D. Lifka, G. D. Peterson, R. Roskies, J. R. Scott, N. Wilkens-Diehr, Xsede: Accelerating scientific discovery, Computing in Science and Engineering 16 (2014) 62-74. 


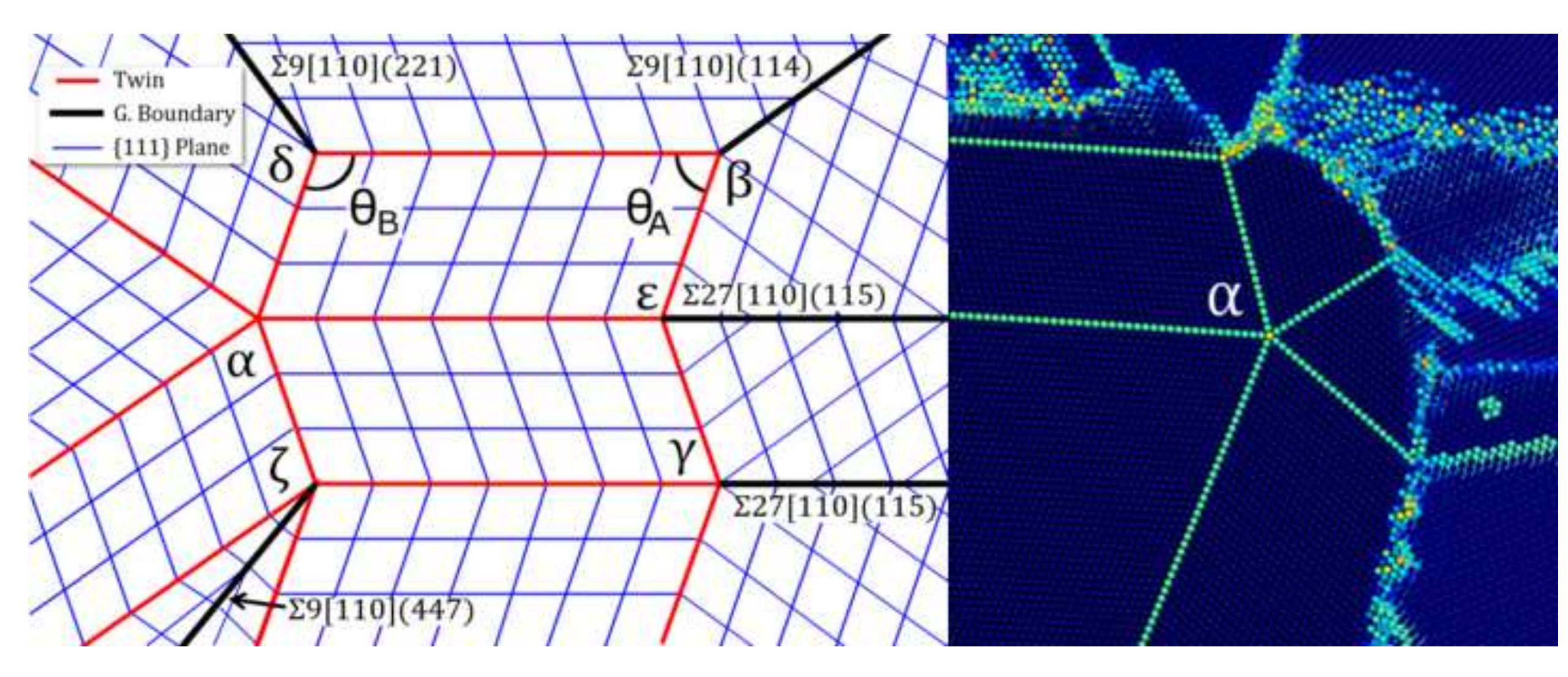




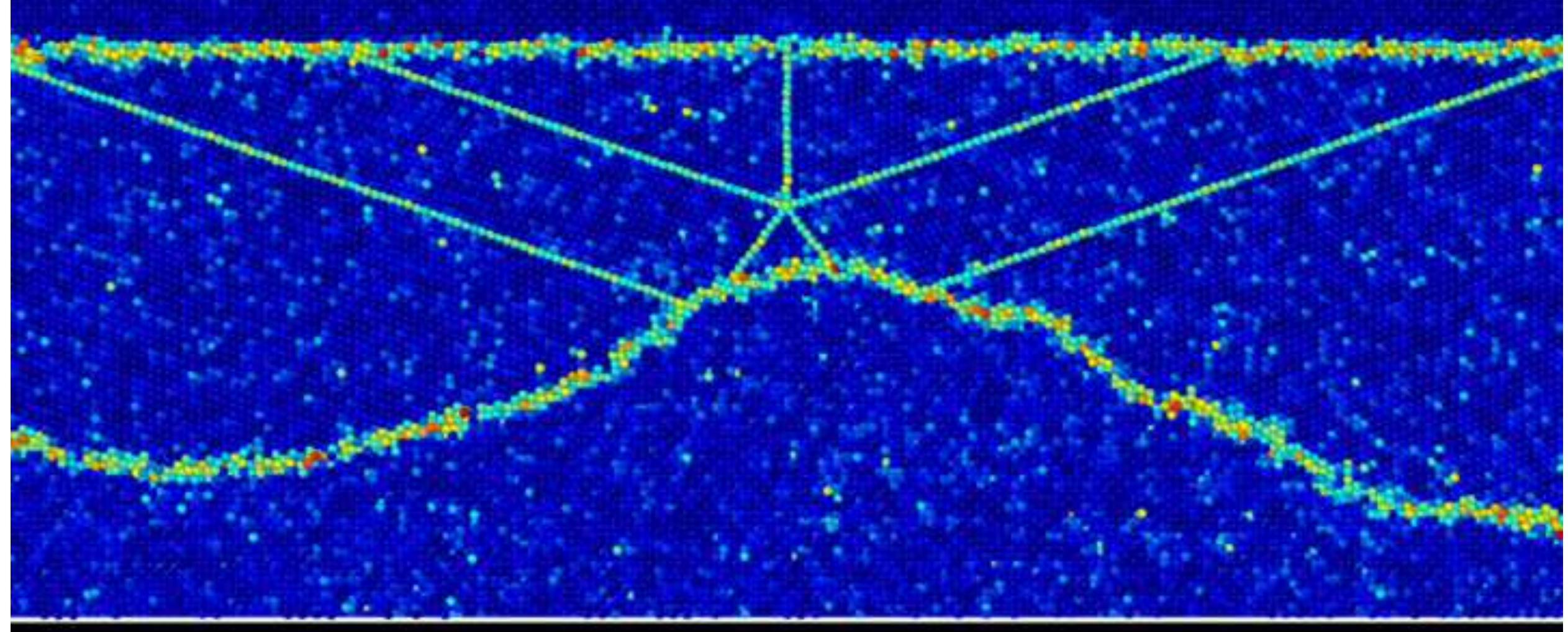

